\title{
CLUN: Concentración por fusión de tres cooperativas lácteas en el cooperativismo agrario en Galicia
}

\author{
María Bastida Domínguez \\ Ana Olveira Blanco \\ Maíte Cancelo Márquez
}

RESUMEN: El sector lácteo gallego, pese a ser un sector estratégico, se ha caracterizado tradicionalmente por un minifundismo que puede restar potencial competitivo a las empresas que operan en él -muchas, bajo fórmula cooperativa-. En este trabajo se describe el proceso de concentración de tres cooperativas lácteas -Feiraco, Os Irmandiños y Melisanto- a través de la creación de la cooperativa de segundo grado Unión de Cooperativas Lácteas Unidas (CLUN). Este proceso, junto a la actual fusión pilotada por la cooperativa AIRA, contribuye a redibujar el cooperativismo agrario y el sector lácteo en Galicia.

El estudio se inicia con una breve descripción del sector agrario, y más en concreto lácteo, en Galicia, contextualizándolo en la evolución del sector en España. A continuación se describe el proceso de creación de CLUN, como ejemplo de concentración en el cooperativismo agrario gallego. Se describen las diferentes entidades que confluyen en la organización resultante, incluyendo las diferentes perspectivas del proceso. Por último, se identifican los retos que debe afrontar CLUN a medio plazo, para finalizar con un conjunto de conclusiones y recomendaciones que identifican un conjunto de buenas prácticas que pudieran contribuir al éxito en este y otros procesos de concentración similares. A este respecto, debe señalarse que el 1 de enero de 2019 CLUN se conforma como cooperativa de primer grado, consolidándose la fusión de las tres cooperativas. Esto sugiere que el proceso de reordenación del cooperativismo agrario en Galicia sigue muy activo, lo que redunda en el atractivo de su seguimiento como objeto de análisis.

PALABRAS CLAVE: Cooperativas agrarias, Economía Social, fusión, CLUN, Galicia, concentración empresarial, sector lácteo.

CLAVES ECONLIT: D22, D61, Q130, J54.

Cómo citar este artículo / How to cite this article: BASTIDA, M., OLVEIRA, A. \& CANCELO, M. (2019): "CLUN: Concentración por fusión de tres cooperativas lácteas en el cooperativismo agrario en Galicia", CIRIEC-España, Revista de Economía Pública, Social y Cooperativa, 96, 29-64. DOI: 10.7203/CIRIEC-E.96.12915.

Correspondencia: María Bastida, Departamento de Organización de Empresas y Comercialización de la Universidad de Santiago de Compostela, maria.bastida@usc.es; Ana Olveira, Doctoranda del Departamento de Organización de Empresas y Comercialización de la Universidad de Santiago de Compostela, y Maite Cancelo, CECOOP, Centro de Estudios Cooperativos de la Universidad de Santiago de Compostela. 


\section{EXPANDED ABSTRACT}

\section{CLUN: A business-case of concentration by merger of three dairy cooperatives at Galicians agrarian cooperatives}

\section{Objectives}

Despite being a strategic sector of Galician's economy, the dairy sector has been traditionally characterized by its small-scale. This factor can inhibit the competitive potential of the companies operating in this area, mainly cooperatives. This paper provides an overview of the steps taken towards the consolidation of a second-degree cooperative known as CLUN -Cooperativas Lácteas Unidas. This process, taken together with a new merger (Aira, SCG) redefines the agrarian cooperative sector as well as the Galician dairy one.

We start by providing a brief analysis of the dairy sector in Spain and Galicia to depict the general context in which this study is undertook. Then, we outline the setting up of CLUN as a concentration process on the Galician agrarian sector. We analyze both the former companies which jointly manage the second-degree one, and the different stages carried out. Next, we identify the major remaining changes to be resolved on the medium term and the main conclusions of the process. Finally, we set up several recommendations regarding the success of this process and other similar consolidations.

Taken this into account, our main objective is to further the study in the role of the size and the concentration processes on the competitiveness of cooperatives, shedding light on the main factors that can might improve -or inhibit- these processes. In sum, we aim to analyze the threats and opportunities that mergers generate when are implemented, and to provide information about some factors that can improve the opportunities towards a successful concentration.

\section{Method}

In order to accomplish our goals, we used a mixed method. First, a review of the main indicators of the agrarian cooperatives -both in Spain and Galicia- has been conducted. This enables us to contextualize our study. Second, we conducted a broad review on the academic literature related to this field, focusing on both those papers that highlight the importance of the size of cooperatives on competitiveness and those that carried out research on concentration processes. Finally, we introduce some issues that suggest the convenience of using a qualitative approach. More specifically, we aim to obtain some information about the business-case as perceived by its internal stakeholders. In this 
respect, some managers were interviewed to identify the main factors they found interesting related to the topic.

\section{Results}

Since the first consolidated income statement has not been approved to date, it is early to determine the financial success of the integration process. However, it is possible to identify some of the factors that have been traditionally related to the competitiveness of agro-food companies: size, diversification and market orientation. Therefore, it might be suggested that the CLUN's creation represents a daring commitment to improve the competitiveness on the dairy sector.

In addition, we can highlight several practices that seem to favor the implementation. Some of them are related to the idiosyncrasy of the organizations, while others arose as a result of the procedures implemented along the integration. In the first place, although the three former companies were linked on their corporate object, the different specialization's focuses make them complementary. Thus, Feiraco plays a leading role in marketing and distribution; Os Irmandiños in the manufacture of feed and Melisanto has an important portfolio of services for its members. This complementarity helped, for example, to avoid the transfer of personnel and to facilitate the establishment of the decision centers at headquarters.

Secondly, we would like to highlight the background on organizational knowledge that the three cooperatives had among themselves, since they had previously shared business in recent years. This mutual knowledge leads to improve the opportunities to undertake merger' processes in a natural way and to overcome in turn possible frictions.

This circumstance, taken together with the coincidence on the company kind and also with the observance of the principles of the Corporate Governance within cooperatives, seems to suggest that they have similar business cultures. This factor is crucial for the success of any concentration process, since that in addition to the financial and organizational adjustment social alignment is essential. Therefore, the combination of a similar business culture and a coordinated management team can help in the successful accomplishment of the integration. However, we claim the need to advance in the equalization of rights, obligations and benefits among workers, in order to reduce some risks in the integration.

Finally, it should be noted that there was a common choice of the external and independent stakeholder for the realization of the valuation proposal and subsequent determination of the percentage of participation of each organization in the resulting entity. In this way, a process that could be a source of conflict is carried out in a widely accepted way. 
In future research it would be interesting to analyze whether these factors could be hierarchized, and also if there are significant differences between the different stakeholders that have participated in the concentration. Namely, if managers of cooperatives have opinions that are statistically different from those provided by workers or external stakeholders. In addition, it could be explored whether types of factors could be established. For instance, social, organizational and financial ones.

\section{Contribution}

This work helps to increase the existing evidence about concentration processes in cooperatives. This kind of business have proven to be a successful way to reduce the unemployment. In addition, they have showed to be able to maintain a certain amount anticyclic behavior in order to survive on financial crisis. But to improve their opportunity of succeeding, they still need to implement strategies to favor their competitiveness. Our results show that effort has to be placed mainly in concentration but keeping in mind that It is important for members to learn how to handle problems and potential clashes using the right practices. It should be noted that CLUN operates as a first-degree cooperative since January of 2019. This suggests that these corporate restructuring processes seem to be very active in Galicia, which highlights the convenience of monitoring them. Further initiatives int this respect might raise the level of consciousness towards the importance of societal dimension to sharpen the competitiveness of companies working in this field.

KEYWORDS: Cooperatives, Social Economy, merger, CLUN, Galicia, business concentration, dairy sector. 


\section{CLUN: CONCENTRACIÓN POR FUSIÓN DE TRES COOPERATIVAS LÁCTEAS EN EL COOPERATIVISMO AGRARIO EN GALICIA}

\section{Introducción}

En 2017 Galicia contaba con 169 cooperativas agrarias, un 5,32\% del total de las cooperativas de este sector registradas en este año en el conjunto del Estado ${ }^{1}$. La comunidad gallega ocupa el octavo puesto en relación con el resto de las comunidades autónomas, lejos de las que cuentan con el mayor número de entidades de este tipo. Concretamente, en 2017 las comunidades con mayor peso en el cooperativismo fueron Andalucía, Castilla-La Mancha, Castilla y León y la Comunidad Valenciana, que aportan conjuntamente el 57,32\% del total de cooperativas españolas. En 2017 el cooperativismo agrario representaba en Galicia el 30,61\% de las cooperativas activas en la comunidad, con una facturación que el año precedente ${ }^{2}$ superaba los 1.880 millones de euros (un 6,5\% de la facturación total de las cooperativas agrarias en España).

El cooperativismo agrario está presente en Galicia, al igual que en el resto del Estado, desde principios del siglo XX, enraizado en las sociedades y sindicatos agrarios. Desde su origen es posible detectar dos características distintivas: el reducido tamaño de las organizaciones y su papel como agente de modernización del sector (Barreiro Gil, 2013). La primera de las características se relaciona con el régimen predominante de propiedad de la tierra en esta comunidad, con un claro protagonismo del minifundismo. La segunda permite situar el cooperativismo como agente impulsor en la modernización productiva de una agricultura adaptativa (Barreiro Gil, 2013; Jordán Rodríguez, Coutinho Villnueva, y Mougán Bouzón, 2004).

En contraste con el panorama general de pequeñas cooperativas, dos grandes entidades -Feiraco, S. Coop Galega y Coren, S. Coop Galega- han sido figuras clave en el avance reciente del cooperativismo agrario en Galicia (Mougán Bouzón, 2014), lo que permite inferir la importancia del tamaño de las cooperativas como factor determinante de su crecimiento y sostenibilidad. En este contexto se enmarcan dos fenómenos recientes que vienen a redibujar el cooperativismo agrario y el sector lácteo en Galicia: la creación de la cooperativa de segundo grado Cooperativas Lácteas Unidas - CLUN, S. Coop Galega- que comienza su funcionamiento el 1 de enero de 2017; y el nacimiento de Aira, Sociedade Cooperativa Galega justo un año más tarde 3 .

1.- Datos Estadísticos de Economía Social. Ministerio de Trabajo, Migraciones y Seguridad Social. Existen numerosas fuentes de datos en relación con las empresas cooperativas (Observatorio Socioeconómico del Cooperativismo Agroalimentario Español -OSCAE- o los diferentes servicios estadísticos de las diferentes comunidades autónomas). Entre una y otras fuentes existen diferencias importantes, como también retardos en la información. Por ejemplo, el último informe disponible del OSCAE es de 2018, con datos de 2016. Además, el tratamiento y categorización de las cooperativas difiere entre las distintas comunidades. Por ello, en este trabajo se utilizan los datos facilitados por el MTMySS, en función de los datos sobre sociedades cooperativas recogidos en las estadísticas que elabora anualmente. ponible.

2.- Datos obtenidos del Observatorio Socio-económico del Cooperativismo Agroalimentario Español, OSCAE. 2016 es el último año dis-

3.- Aira Sociedade Cooperativa Galega procede de la fusión de las cooperativas Aira (Taboada), Agris (Palas de Rei), Coelplan (de Becerreá), Cogasar (Sarria) e lcos (de Chantada), que funcionan desde el 1 de enero de 2018 como una única entidad. Aira está integrada por 2.574 productores, con una cifra de negocio que ronda los 90 millones de euros, gracias a la producción de 300 millones de litros de leche al año y unas 200.000 toneladas de pienso. 
El objetivo principal de este trabajo es analizar y describir de forma detallada el proceso de concentración de estas cooperativas, con particular atención a los factores que lo impulsaron, las necesidades que viene a cubrir, y los retos a los que la nueva entidad debería tratar de dar respuesta. Para ello, en primer lugar, se establece el marco general de análisis, introduciendo la situación del sector agrario -y concretamente, el lácteo- en España. A continuación, se sitúa el foco en el entorno más próximo, desarrollando un breve análisis del sector en Galicia que permite determinar las condiciones socioeconómicas que acompañan el proceso de integración. En este contexto, se prestará especial interés a la importancia de la dimensión de las organizaciones en este sector, determinando cómo esta situación afecta a la competitividad ante mercados cada vez más globales y consumidores más exigentes.

Una vez realizada esta contextualización, se describe el nacimiento de CLUN como proceso de concentración en el cooperativismo agrario gallego. Se introducen las diferentes entidades que participan en la organización resultante, describiendo sus actividades y analizando sus datos socioeconómicos con el fin de identificar las causas internas que determinaron el proceso de concentración. Con este objetivo se analizan los datos incluidos en los diferentes registros de cuentas existentes en los registros de cooperativas de los ejercicios 2014, 2015 y 2016. Este análisis se completa con la información obtenida de dos entrevistas semiestructuradas realizadas a las responsables de los departamentos de Recursos Humanos y Marketing de la cooperativa Feiraco.

Finalmente, se destacarán los retos que CLUN debe afrontar a corto plazo, así como una serie de conclusiones y recomendaciones extraídas del proceso de concentración. Se sugiere también un conjunto de buenas prácticas que pudiera determinar el éxito en este y otros procesos de concentración.

\section{Contexto Socioeconómico}

El proceso que nos ocupa se inscribe en un contexto concreto que será objeto de breve análisis en este epígrafe. A continuación, se destacan los puntos fundamentales del cooperativismo agrario, el sector lácteo, la importancia de la dimensión de las organizaciones en este sector, y el marco normativo que lo regula.

\section{a. El cooperativismo agrario}

El fin de la dictadura franquista en 1975 supuso un cambio socioeconómico para España que afectó también a las sociedades cooperativas. Así, a finales de la década de los 70 se produce un 


\section{CLUN: CONCENTRACIÓN POR FUSIÓN DE TRES COOPERATIVAS LÁCTEAS EN EL COOPERATIVISMO AGRARIO EN GALICIA}

incremento notable en la aparición de nuevas cooperativas agrarias, en línea con la evolución del cooperativismo agrario que se produce al mismo tiempo que en el resto de Europa. Entre los años que transcurren entre 1975 y 1983, según los datos del Comité Europeo de Cooperativas Obreras de Producción (CECOP) el número de cooperativas en España pasó de 8.000 a 22.000 (Morales, Romero y Muñoz, 2003). Este dinamismo continúa hasta 1986, año en que el número de cooperativas creadas en España alcanza las 2.746. Durante estos años proliferan una serie de medidas de apoyo legislativo y financiero a este tipo de organizaciones, que les permite servir como instrumento clave de lucha contra el desempleo (Morales et al., 2003). No obstante, en la década de los 90 esta tendencia no solo se ralentiza, sino que se invierte, con lo que el número de cooperativas activas en España pasa de 23.697 a principios de la década a situarse alrededor de 17.000 en 1999, una reducción superior al $39 \%$.

A partir del año 2001 la competitividad de la agricultura europea se ve afectada por nuevos factores como la introducción de tecnología avanzada, la innovación en la gama de productos, la elevación del nivel de cualificación del capital humano y la mejora de las estructuras empresariales y organizativas. Ello favorece la irrupción de un nuevo escenario agroalimentario en el que algunas cooperativas agrarias comienzan a integrarse con un alto grado de participación. Se exigen también mejoras en la calidad de los productos y como resultado, diferentes asociaciones del sector - como por ejemplo Cooperativas Agroalimentarias de España- se plantean la posibilidad de introducir cambios en modelos de producción.

La tabla 1 nos muestra la evolución del número de cooperativas en España y Galicia, tanto el total como las cooperativas agrarias, desde el año 2000 hasta 2017, último dato disponible en los registros oficiales ${ }^{4}$. Se incluye también el número de trabajadores en estas sociedades. 


\section{Tabla 1. Cooperativas Totales y Agrarias 2000-2017 (número y trabajadores), Galicia / España}

\begin{tabular}{|l|r|r|r|r|r|r|r|r|r|r|r|}
\hline \multicolumn{5}{|c|}{} & \multicolumn{5}{|c|}{ No COOPERATIVAS } & \multicolumn{5}{c|}{$N^{\circ}$ TRABAJADORES } \\
\hline \multirow{2}{*}{ Total } & 2000 & 2005 & 2010 & 2015 & 2017 & 2000 & 2005 & 2010 & 2015 & 2017 \\
& España & 17.037 & 16.391 & 13.310 & 11.958 & 12.056 & 204.490 & 237.927 & 226.165 & 220.359 & 233.309 \\
& Galicia & 681 & 675 & 595 & 579 & 552 & 7.567 & 7.017 & 6.747 & 6.452 & 6.235 \\
\hline \multirow{2}{*}{ Agrarias } & España & 4.118 & 3.659 & 3.487 & 3.292 & 3.225 & 48.437 & 68.413 & 67.013 & 65.177 & 66.637 \\
& Galicia & 168 & 213 & 201 & 187 & 169 & 1.393 & 1.371 & 1.395 & 1.381 & 1.361 \\
\hline
\end{tabular}

FUENTE: Elaboración propia con datos estadísticos de Economía Social (MTMySS).

Se puede comprobar que el número de cooperativas en España desciende progresivamente en los últimos años, con un ligero repunte en las cooperativas totales en España en 2017. En Galicia, la tendencia es similar, aunque también este último período mantiene signo negativo. No obstante, cabe destacar que el ritmo de decrecimiento promedio del número de cooperativas en España desde el año 2.000 (2,01\% en promedio anual) es superior al dato gallego (1,42\%). De forma similar, mientras que en España las cooperativas agrarias descienden a una media del 1,24\% anual, en la comunidad gallega se registra un leve incremento del $0,01 \%$. Se observa también que el promedio de trabajadores por cooperativa es menor en Galicia que en España. Así, en el período contemplado las cooperativas españolas emplean a una media de 1,63 trabajadores, frente a los 1,11 de promedio en las cooperativas gallegas. En el caso particular del cooperativismo agrario la diferencia es mayor: la media de trabajadores empleados en España en este sector (1.803) más que duplica a los empleados en Galicia (742). Esta circunstancia parece sugerir un menor tamaño de las cooperativas agrarias gallegas.

El gráfico siguiente nos permite observar la evolución anual en la creación de nuevas cooperativas en España y Galicia en el mismo período. 


\section{Gráfico 1 a. Cooperativas Creadas 2000-2017, Galicia / España}

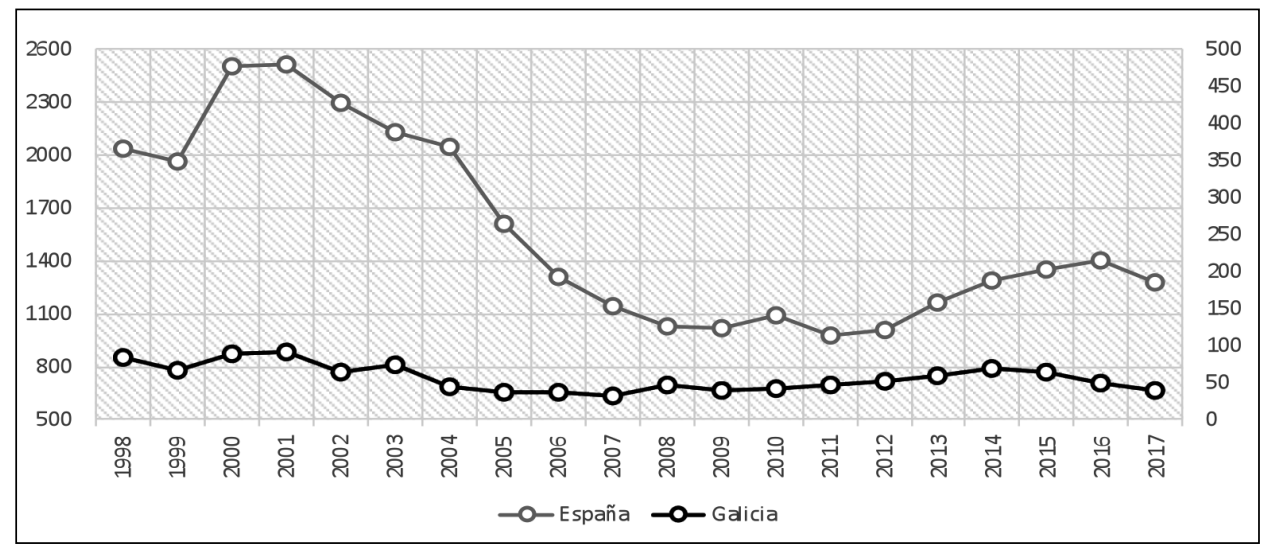

FUENTE: Elaboración propia con datos estadísticos de Economía Social (MTMySS).

Se observa cómo los períodos de bonanza económica coinciden con aquellos en los que desciende el número de cooperativas creadas, mientras que aumenta durante la reciente crisis económica (20072013), A partir de 2015-2016 se ralentiza la creación de sociedades cooperativas, que vuelve a caer en 2017. Cabe destacar que este carácter anticíclico responde al patrón establecido en estudios precedentes que asocian la creación de cooperativas con etapas de recesión económica y desempleo, y su disminución con épocas de recuperación o crecimiento económico (Bretos, Díaz-Foncea y Millán, 2018).

Aparentemente, los datos sugieren una mayor estabilidad en el comportamiento de las cooperativas gallegas. No obstante, si completamos la información con el análisis de la tasa de variación de las cooperativas creadas anualmente (Gráfico 1.b), esta impresión cambia. 


\section{Gráfico 1 b. Tasa de variación anual en la creación de cooperativas 2000-2017, Galicia / España}

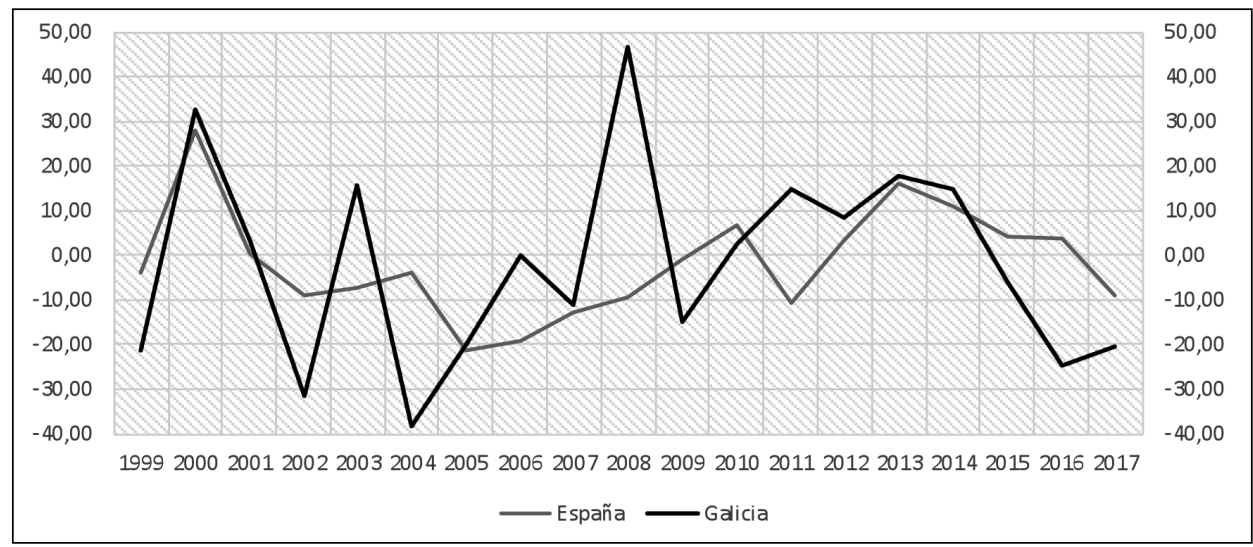

FUENTE: Elaboración propia con datos estadísticos de Economía Social (MTMySS).

Se puede comprobar que las tendencias son similares, aunque, al contrario de lo sugerido previamente, la oscilación tiene más fuerza en la comunidad gallega. La creación de cooperativas aumenta en Galicia cuando lo hace en España, y cae también en los mismos períodos salvo el bienio 20082009 y el 2010-2011. Asimismo, mientras en Galicia disminuye con fuerza el número de cooperativas creadas anualmente a partir de 2015 , esta circunstancia es más atenuada a nivel estatal, pasando a valores decrecientes el pasado 2017. En concordancia con la relación antes apuntada entre la situación económica general y la creación de cooperativas, debe apuntarse que la crisis económica en Galicia tuvo efectos con cierto retardo en relación con la economía española; como también los indicadores económicos se tornaron positivos con cierto adelanto, a partir precisamente de 2015.

Por otra parte, como avanzamos en la introducción el cooperativismo en España muestra una alta concentración en determinadas comunidades autónomas. El protagonismo de cada comunidad en el cooperativismo en general no coincide con el peso relativo de cada una de ellas para el caso de las agrarias. Así, a pesar de que Andalucía lidera ambas clasificaciones, mientras que en el porcentaje total de cooperativas Cataluña y Valencia le siguen en importancia con valores superiores al $10 \%$ de las cooperativas en el conjunto del estado, en las agrarias estas comunidades ceden su liderazgo en favor de Castilla-La Mancha y Castilla y León. El análisis también difiere a la hora de examinar la importancia relativa que las cooperativas agrarias tienen en el tejido cooperativo de cada comunidad. La Rioja y Castilla-La Mancha destacan con claridad en este análisis, habida cuenta de que más del $50 \%$ de las cooperativas en estos territorios son agrarias.

El gráfico 2 permite comprobar cómo Andalucía lidera el peso del cooperativismo, tanto general como agrario, en el año 2017: 


\section{Gráfico 2. Distribución de Cooperativas en España (CC.AA), 2017}

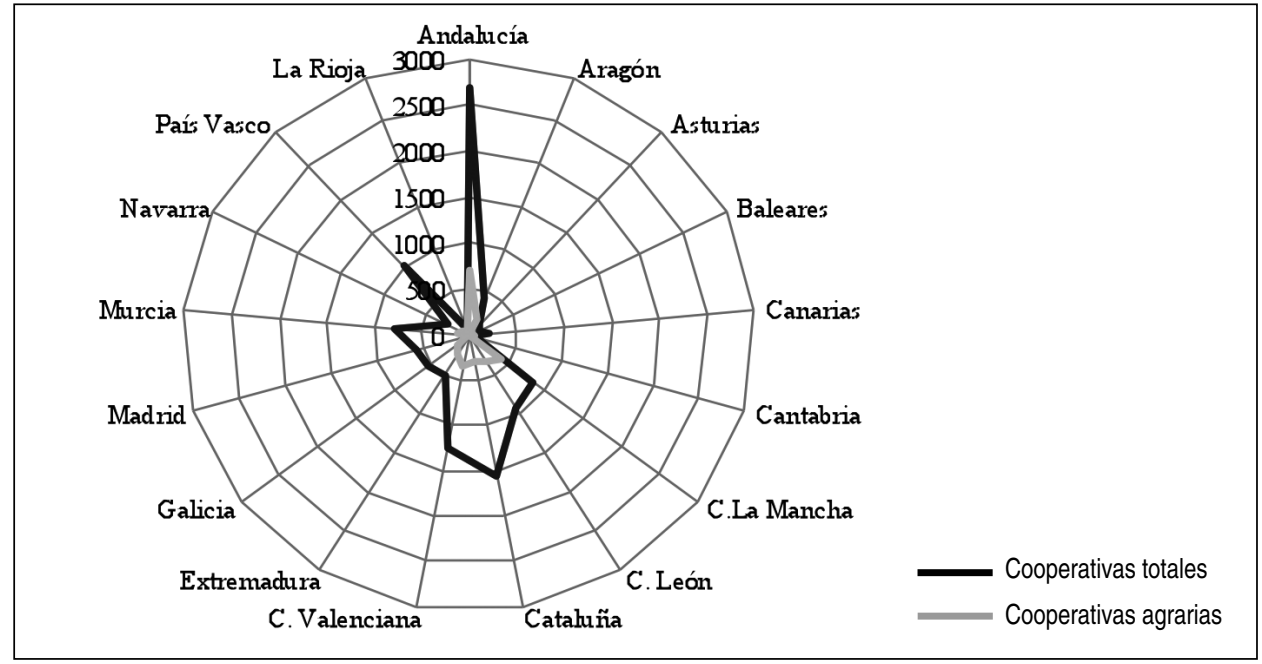

FUENTE: Elaboración propia con datos estadísticos de Economía Social (MTMySS).

Galicia, donde el 30,62\% de las cooperativas activas en 2017 son agrarias, aporta el 4,58\% de las cooperativas del estado y un $5,32 \%$ de las agrarias, así como el $2,67 \%$ de los trabajadores empleados en estas sociedades (2,04\% de los empleados en cooperativas agrarias). Cabe señalar que estos valores son inferiores a la participación de las empresas gallegas en el total estatal (6\%), como también a su peso relativo en la población activa $(5,45 \%)$ y ocupada $(5,95 \%)^{5}$.

El sector agrario no se ha mantenido al margen de la crisis económica, con el agravante del efecto que la volatilidad de los precios de mercado tuvo sobre el mismo, agudizando los problemas económicos y financieros. Todo ello derivó en una situación extremadamente difícil para la mayoría de los productores. Por ejemplo, en el año 2009 la renta agraria media de la Unión Europea caía un 12\% (Gómez, 2015). Otras de las graves consecuencias que sufrió el cooperativismo, al igual que el resto del tejido societario, fue el descenso de demanda interna, lo que impulsa la salida a mercados exteriores como refugio y vía de subsistencia. Así, a partir de 2010 aumentaron las exportaciones de productos lácteos a la UE, mejorando notablemente la balanza comercial en este sector 6 .

Este es, a grandes rasgos, el marco que el cooperativismo agrario presenta a CLUN, S. Coop Galega en el momento de su gestación y nacimiento el 1 de enero de 2017. Pasamos a continuación al análisis del sector específico de actuación de la cooperativa, el sector lácteo. 


\section{b. El sector lácteo}

El sector lácteo en España produce más de 7 millones de toneladas de leche al año con un aumento constante en los últimos ejercicios. Así, según los datos registrados en la Subdirección General de Productos Ganaderos de la Dirección General de Producciones y Mercados Agrarios, las entregas de leche cruda registraron un aumento de 133.841T entre 2016 y 2017, cerca de un $2 \%$. El sector lácteo gallego ha evolucionado en las últimas décadas con notable dinamismo. Así, las entregas a la industria pasan de 1.206 millones de litros en 1985 a 2.520 en 2016, y la producción de leche se incrementó un 1,7\% en el mismo período. En concreto, la aportación de la comunidad gallega al total de entregas de leche estatal en 2017 triplica la correspondiente a Castilla y León y Cataluña, segunda y tercera productora respectivamente. Este nivel de producción próximo a las 2.600.000T coloca a Galicia entre las diez primeras regiones lácteas de la Unión Europea?.

\section{Gráfico 3. Entregas de Leche Cruda (2017)}

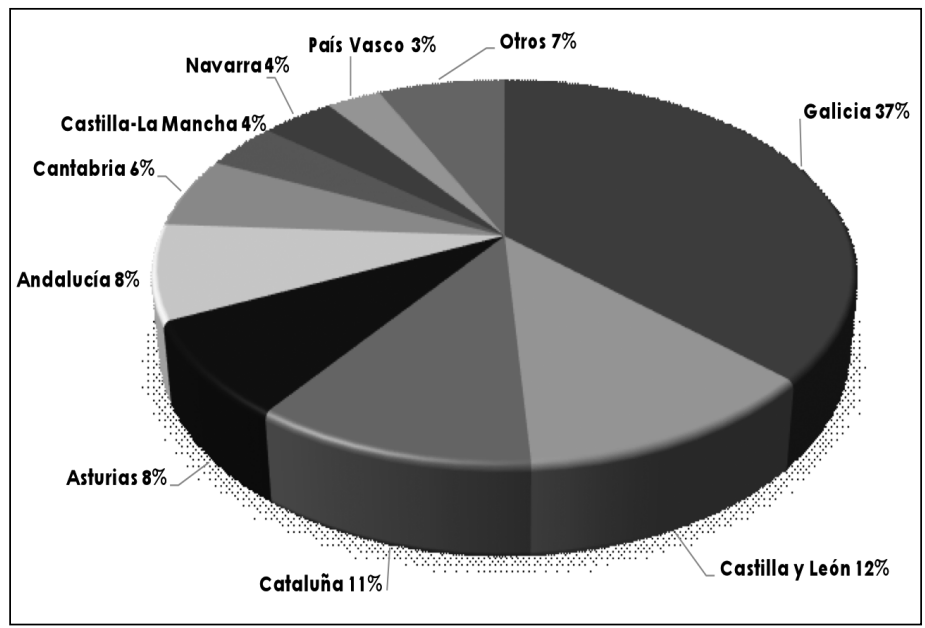

FUENTE: Elaboración propia con datos del Misterio de Agricultura, pesca y alimentación (Subdirección General de Productos Ganaderos Dirección General de Producciones y Mercados Agrarios, 2018).

Los datos cambian si el foco de análisis se sitúa en el censo de ganado vacuno y el número de explotaciones. En este caso, la tendencia es decreciente conforme avanza el tiempo. El número total

7.- Los últimos datos disponibles (Mayo 2018) refuerzan estas tendencias. Así, mayo de 2018 registra un incremento del volumen de entregas del 1,7\% interanual para el conjunto del Estado, que se duplica en Galicia (3,7\%). 
de ganaderos con entregas declaradas de leche en España es de 14.184 para el mes de mayo de 2018, un descenso $6 \%$ en relación con el mismo mes del año anterior. En la comunidad gallega el sector ha acelerado su reestructuración, con una reducción del número de explotaciones de 100.000 a 9.000 (Sineiro García, López Iglesias y Santiso Blanco, 2017) desde 1985. Galicia aporta el 55\% del total de ganaderos y ganaderas en el conjunto del Estado, lo que permite intuir el pequeño tamaño de las explotaciones gallegas, como se aprecia en el gráfico $4^{8}$. Le siguen Asturias (13\%), Cantabria y Castilla y León ( $9 \%$ y $8 \%$ del total, respectivamente).

\section{Gráfico 4. Ratio Entregas de leche / $\mathbf{n}^{\circ}$ ganaderos por C. Autónoma (2018)}

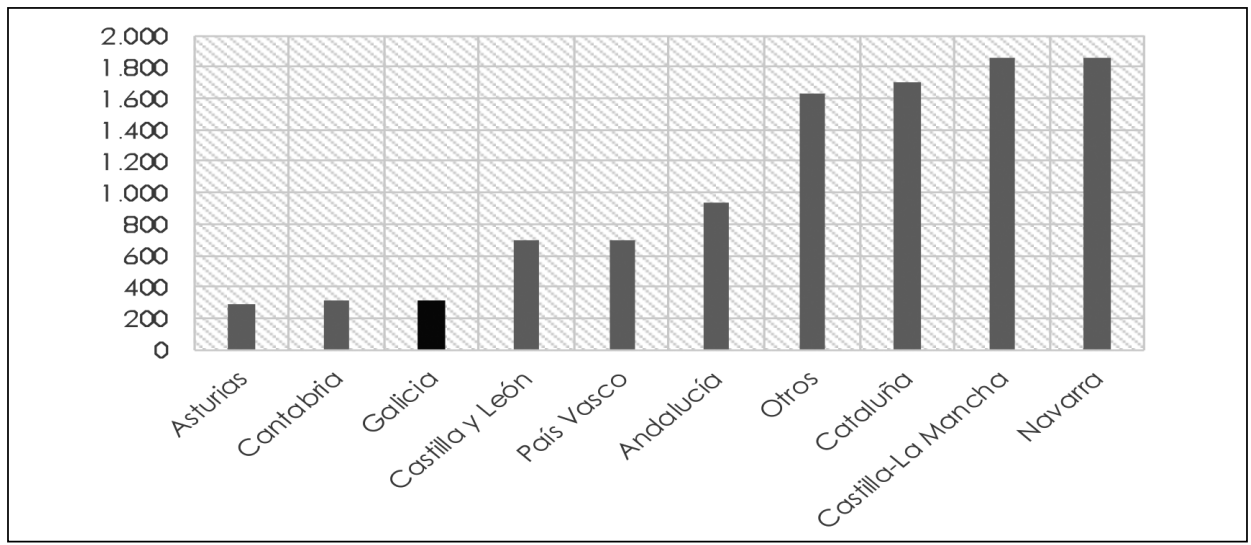

FUENTE: Elaboración propia con datos de FEGA (2018).

En el año 2013 se producen dos acontecimientos fundamentales que van a afectar al sector agrario a nivel europeo y español: los Acuerdos de la reforma de la PAC y la aprobación de dos textos legislativos, la Ley de Mejora de Integración Cooperativa y otras Entidades Asociativas y la Ley de Mejora del Funcionamiento de la Cadena Alimentaria. El primero, que culminará con la abolición de la cuota láctea en 2015, afecta de forma destacada al mercado de productos lácteos, determinando la necesidad de adaptación de las cooperativas a una nueva política de producción. Esto acarrea diferentes problemas para los productores y cooperativas que aún a día de hoy intentan solventar, siendo lo más eficientes posibles sin perder su participación en el mercado, tanto español como europeo. Esta reforma, junto con la globalización y los cambios en la demanda, configuran un contexto de retos para

8.- Así, nos encontramos que entre las explotaciones de dimensión mediana y grande -muchas de ellas bajo fórmula cooperativa- el $65 \%$ pertenecen a la Orientación Técnico-Económica (OTE) bovino de la leche. 
las cooperativas agrarias (Juliá et al., 2013; Lájara-Camillieri y Server-Izquierdo, 2017) que en el sector lácteo se ven potenciados por (Sineiro García y otros, 2017):

- El incremento da volatilidad de los precios de la leche en la UE.

- La vinculación de esos precios con las oscilaciones de los productos industriales en los mercados internacionales.

Galicia, donde los precios ${ }^{9}$ por lo general no alcanzan la media española y europea, se vio más afectada por estos factores, con un conjunto de crisis recurrentes desde la entrada de España en la Unión Europea en los años ochenta. La última, en 2015, acuciada por una fuerte reducción en los precios y falta de garantías en la recogida de la producción (López Iglesias, 2015).

En lo que se refiere al peso del sector en el PIB de Galicia, en 2014 el sector lácteo aporta el 1,85\% del PIB total ${ }^{10}$, cifra superior al peso económico del sector textil y confección $(1,1 \%$ y próximo al de la industria del automóvil (2,8\%). No obstante, debe destacarse que estas cifras esconden una debilidad estructural, puesto que la existente está orientada a derivados simples y de escaso valor añadido. De hecho, si analizamos el Valor Agregado Bruto (VAB) encontramos que en Galicia en $81 \%$ procede de las explotaciones y la industria solo aporta 24 céntimos, seis veces menos que el caso de Cataluña o Asturias. Además, sólo el $30 \%$ de la materia prima gallega se transforma en industrias locales (López Iglesias, 2015; Sineiro García y otros, 2017).

Estas cifras pueden explicarse parcialmente atendiendo a la realidad del tejido productivo subyacente. En este contexto, las explotaciones gallegas tienen una reducida base territorial y una creciente dependencia de alimentos comprados, lo que las hace muy vulnerables a cualquier tipo de variación que se produzca en los precios de los cereales. Coexisten dos grandes tipos de cooperativas: las que actúan como simples intermediarias comprando la leche a sus socios, y las cooperativas industriales ${ }^{11}$. Estas últimas, entre las que se sitúan las que componen nuestro objeto de estudio, si alcanzan una gran dimensión y lideran el sector en las zonas en las que se implantan, son fundamentales para la sostenibilidad; pero si no se alcanza esa dimensión se encuentran en una situación de desventaja frente al resto ${ }^{12}$. Comenzamos ya a intuir la importancia de la organización como factor que determina la competitividad, que discutimos más ampliamente en el siguiente apartado.

9.- El precio medio de leche en Galicia en abril de 2017 fue de 30,80 c/l, un céntimo por debajo del registrado en España en el mismo período $(31,80 \mathrm{c} / \mathrm{l})$.

10.- Estimación realizada por la asociación "Terra e Leite" en su documento "21 Propostas de Futuro" (2017).

11.- En Galicia existen registradas en la Asociación Galega de Cooperativas Agroalimentarias 34 cooperativas dedicadas al sector del vacuno. La Asociación pretende fomentar que las pequeñas cooperativas trabajen conjuntamente y se fusionen con otras para dar más protagonismo a nuestra Comunidad Autónoma en la producción y distribución de productos lácteos.

12.- Según el documento $n^{\circ} 12$ de la asociación Galega Terra e Leite titulado: "A situación do sector lácteo. Apuntes para a reflexión e o debate", http://terraeleite.org/documentos/a-situacion-do-sector-lacteo-apuntes-para-a-reflexion-e-o-debate/. 


\section{c. La dimensión como factor determinante para afrontar nuevos retos}

Como vimos en el análisis del entorno, ante la disminución de la demanda interna, la opción de una buena parte de las organizaciones fue reforzar la internacionalización 0 acudir por primera vez al mercado exterior para intentar dar salida a una producción que no dejaba de crecer (Flecha y Ngai, 2014; Siebel, 2016). Esta realidad la recoge la Asociación Gallega de Cooperativas -AGACA- cuando presenta su XVIII Informe sobre la gestión y el estado del cooperativismo: "se está consolidando una nueva forma de actuar centrada en la perspectiva global e internacional". Simultáneamente, se establece la necesidad de mejora de la dimensión de las organizaciones como condición necesaria para mejorar la competitividad, aumentar el valor añadido y satisfacer las necesidades de las personas que forman las cooperativas y de los clientes (Mougán Bouzón, 2014).

Las cooperativas se han caracterizado tradicionalmente por su pequeño tamaño, vinculado a su enraizamiento socioeconómico y territorial (Bretos y otros, 2018). Esta realidad de atomización es común al conjunto del cooperativismo agrario español, puesto que no puede desvincularse de la dispersión geográfica y la atomización de la propiedad, y se traduce en debilidad al llevar pareja una escasa capacidad negociadora frente a los restantes agentes del mercado (Campos-Climent y ChavesÁvila, 2012; Juliá y otros, 2012; Meliá Martí y Martínez García, 2015). La necesidad de convergencia determina que en años recientes la legislación otorgue cierta flexibilidad a las diferentes normativas en materias de cooperativas, para facilitar las relaciones entre ellas (Meliá Martin y Server Izquierdo, 2002). Con todo ello, y potenciado también por la creciente globalización económica, las cooperativas han consolidado la tendencia a la concentración (Server Izquierdo y Meliá Martí 2002). Esta tendencia puede inscribirse en la detectada a nivel europeo, si bien es en general en los países del Norte donde se han desarrollado estos procesos con mayor éxito, destacando los ejemplos de Dinamarca, Holanda e Irlanda (Juliá et al., 2015).

Debemos destacar que la relación entre tamaño y rentabilidad, y por ende competitividad, no siempre es exacta. También es cierto que existen otras vías para obtener estos resultados, como la internacionalización para cooperativas que no hacen uso intensivo de capital, la descentralización productiva o la diversificación (Monzón, 2012). Sin embargo, distintos estudios han destacado las ventajas de los procesos de concentración como la obtención de economías de escala, mejora del margen de negociación, posibilidades de diversificación, acceso a nuevos mercados, disminución de riesgos o mejora en las condiciones de acceso a la financiación (Fayos, Calderón y Mir, 2011; Juliá, García, y Meliá Martí, 2012; Juliá, Meliá, García y Gallego, 2010).

En Galicia esta necesidad se ha visibilizado sobre todo desde una perspectiva académica. Así, diversos autores han puesto de relieve la necesidad de terminar con la atomización del sector como vía para que las cooperativas agrarias adquieran mayor peso específico y puedan mejorar su competitividad (Babío Arcay y Jordán Rodríguez, 2008, 2010; Jordán Rodríguez y otros, 2004). 
Las cooperativas, al igual que el resto de las empresas, pueden optar por una concentración empresarial para dar cumplimiento a diferentes tipos de necesidades. En la práctica se corre el riesgo de englobar bajo el término "integración" diversas formas de asociacionismo o cooperación de carácter económico, frente a fórmulas más amplias que engloban diversos vínculos con el objetivo de lograr una representación colectiva para la defensa de intereses heterogéneos. Por tanto, resulta de particular utilidad la propuesta de clasificación de Juliá, Server y Meliá (2004), quienes distinguen las formas de concentración precisamente en función de las necesidades 0 intereses que persiguen: económicas o con finalidad representativa. A su vez, cada uno de estos grupos contiene diferentes subcategorías, como se puede observar en la Tabla 2.

\section{Tabla 2. Formas de concentración empresarial}

\begin{tabular}{|l|l|l|}
\hline Económica & Sin vinculación económica & $\begin{array}{l}\text { Cooperativa de } 2^{\circ} \text { grado } \\
\text { Cooperativa de servicios } \\
\text { Grupos Cooperativos } \\
\text { Otras formas de colaboración económica }\end{array}$ \\
\cline { 2 - 3 } & Con vinculación económica & $\begin{array}{l}\text { Fusión Pura } \\
\text { Fusión por Absorción } \\
\text { Fusión Especial } \\
\text { Escisión } \\
\text { Escisión/Fusión }\end{array}$ \\
\hline Con Finalidad Representativa & Uniones Cooperativas \\
& Federaciones y confederaciones de cooperativas. \\
\hline
\end{tabular}

FUENTE: Adaptado de Juliá lgual et al., 2004.

Tal y como hemos destacado previamente, en las cooperativas lácteas de Galicia pueden destacarse diversas disfunciones que lastran su competitividad como su escasa dimensión, poco margen de negociación, excesiva dependencia de proveedores alimentarios o la baja industrialización. Por tanto, en el caso que nos ocupa puede enmarcarse en el segmento de las concentraciones cuyo origen responde a necesidades económicas. Este, a su vez, permite diferenciar dos grandes grupos en función del grado de vinculación económica entre las entidades participantes, tal y como se observa en la Tabla 2. En el proceso de concentración de CLUN estamos ante una concentración empresarial con motivaciones económicas y sin vinculación patrimonial: una cooperativa de segundo grado.

En España existen en la actualidad 130 cooperativas de segundo grado, que representan el 3,4\% del total de cooperativas agroalimentarias y el $29 \%$ de la facturación total (Meliá-Martí y Peris-Mendoza, 
2017). La cooperativa de segundo grado ha sido considerada históricamente un mecanismo adecuado de integración cooperativa, con una individualidad propia y larga tradición en determinados sectores del cooperativismo. En concreto, ha sido la fórmula de cooperación utilizada preferentemente en las cooperativas agrarias. Esta preferencia puede residir en la tradición normativa y doctrinal, puesto que las diferentes regulaciones, tanto a nivel estatal como autonómico le han otorgado un lugar preferente a la hora de regular los procesos de integración cooperativa. Por otra parte, también debe destacarse que alguno de los principios cooperativos, como el de control democrático, parecen encajar mejor con esta fórmula de integración que con otras más profundas como las fusiones. Asimismo, el sexto principio cooperativo establece que el trabajo en común sirve mejor a los socios y al propio modelo ("cooperación entre cooperativas"). Se trata de una forma de vinculación que modifica el poder de decisión jurídico-económico de las entidades que participan en la misma, a través de la formación de una nueva empresa de titularidad plural que se compatibiliza con el mantenimiento de la independencia jurídica de las sociedades que se agrupan. Su objetivo es la promoción y desarrollo de fines económicos comunes, así como reforzar e integrar la actividad económica de estos. La amplitud de este objetivo, así como el mantenimiento de la personalidad jurídica de las entidades participantes, sugiere cierta flexibilidad en la vinculación entre las sociedades implicadas, así como cierto grado de reversibilidad. Esta flexibilidad implica que este subtipo social corporativo puede alcanzar mayores o menores cuotas de integración en función de la voluntad de sus miembros, desde la mera colaboración empresarial hasta ser un marco organizativo con entidad jurídica propia, respetando siempre la individualidad jurídica de las entidades participantes. Será la autonomía de la voluntad, reflejada en los estatutos, la que determine el modo y alcance de la vinculación.

\section{d. El marco normativo}

Galicia cuenta con competencia exclusiva para el desarrollo y la ejecución de la legislación en materia de entidades cooperativas, tal y como viene recogido en el artículo 28 de la Ley Orgánica 1/1981 del Estatuto de Autonomía de esta comunidad. En ejercicio de dicha competencia, el parlamento gallego promulgó la Ley 5/1998 de Cooperativas de Galicia que en sus artículos 75 y siguientes, recoge los pasos que deben seguirse desde el punto de vista normativo, en los procesos de fusión.

Como primer paso se establece, en el artículo 76, la necesidad por parte de los consejos rectores de las cooperativas participantes de redactar un proyecto de fusión que identifique las cooperativas que van a tomar parte de la operación; el reconocimiento de derechos, participación y obligaciones de los socios -incluyendo el sistema para fijar la cuantía que se reconoce como aportación al capital de la cooperativa nueva 0 absorbente- y la fecha a partir de la cual el acuerdo entra en vigor y actúa a todos los efectos la cooperativa nueva o absorbente.

La fusión debe ser aprobada en asamblea general en cada una de las cooperativas en el plazo de seis meses. La convocatoria de la asamblea en que se somete a votación el acuerdo de integración 
debe ir acompañada de una serie de documentación complementaria, como el proyecto de fusión, los informes de los consejos rectores de cada una de las cooperativas, los balances de pérdidas y ganancias de los últimos tres ejercicios, el de fusión, un proyecto de estatutos y la relación de personas que se propongan para ostentar futuros cargos (art. 77 de la Ley 5/1998).

Además, se establece la necesidad de que el acuerdo sea refrendado por mayoría reforzada. Por tanto, se introducen una serie de requisitos específicos para la validez del acuerdo de integración (art. 78 de la Ley 5/1998): el proyecto de fusión debe ir incluido en la convocatoria de la asamblea general, con reconocimiento expreso de acceso a la documentación por parte de los socios; no puede ser modificado en la asamblea y debe contar con la debida publicidad, mediante su publicación en el Diario Oficial de Galicia y en uno de los diarios de mayor circulación de la provincia en la que tengan su domicilio social cada una de las cooperativas. Toda vez refrendado y publicitado el acuerdo, las cooperativas quedan obligadas a continuar con el proceso.

Como se puede observar, la flexibilización de normas y las iniciativas en el marco de promoción de procesos de integración de cooperativas conviven con toda una serie de requisitos destinados a garantizar la transparencia del proceso, así como los derechos de los socios participantes.

\section{CLUN una nueva realidad en el cooperativismo gallego}

Después de casi dos años de estudios, y siguiendo el ejemplo de otras cooperativas a nivel nacional e internacional, Cooperativas Lácteas Unidas (CLUN) inicia su recorrido el 31 de octubre de 2016 con la ratificación del acuerdo de integración por amplia mayoría de los socios presentes en las asambleas generales convocadas por las tres cooperativas socias: Feiraco, Melisanto y Os Irmandiños ${ }^{13}$.

El acuerdo recoge el objetivo principal de la concentración: "Es la vocación de los promotores que este sea el germen de un gran grupo cooperativo que tenga la dimensión suficiente para dar respuesta a los retos que un mercado cada vez más amplio y competitivo demanda". Esta declaración de principios refleja la necesidad de superar las dificultades que el tamaño introduce para la competitividad en el contexto actual. No obstante, la diferente naturaleza de las entidades participantes permite detectar un objetivo subyacente, el acoplamiento de las tres entidades para aprovechar las sinergias, conocimiento y experiencia de cara a contribuir a la sostenibilidad de la producción láctea. Más concretamente:

13.- Una cuarta cooperativa, Xallas, comenzó los trámites de integración, aunque finalmente se retiró de la iniciativa al no aprobarse el proceso de concentración por parte de su asamblea. 
- La integración y protección de los servicios técnicos-económicos, con el objetivo de alcanzar una mayor eficacia de los costes de producción de sus socios y la mejora continua de las explotaciones.

- La optimización de suministros.

- La agregación de volumen para mejorar la cadena de valor láctea y así contribuir a su estructuración.

Abordaremos el análisis a esta nueva realidad del cooperativismo agrario gallego dividiéndolo en cinco apartados: identificación de las entidades participantes, análisis económico previo a la integración, determinación de la participación en CLUN de cada una de las cooperativas socias, análisis organizativo y retos de futuro.

\section{a. Las cooperativas participantes}

Dentro de las entidades de economía social se diferencian dos grandes grupos, atendiendo a su dimensión (Chaves, 1996). El primero está integrado por entidades que, en su proceso de adaptación al entorno, han llegado a conformar auténticos grupos empresariales cooperativos. Son entidades normalmente de gran tamaño y con una cuota de mercado importante. El segundo grupo estaría conformado por entidades más pequeñas que han surgido como consecuencia de la defensa y adaptación al entorno. Son generalmente entidades jóvenes, con un tamaño pequeño o mediano y con una estructura económica por lo general endeble.

Esta división sigue vigente en la actualidad encontrándose, antes del proceso de concentración, las entidades que nos ocupan a medio camino entre ambos grupos. A continuación, describimos brevemente las tres entidades que aprobaron el proyecto de integración.

FEIRACO S. COOP. GALLEGA- Es una empresa cooperativa gallega que produce, recoge y comercializa leche de vaca y productos derivados procedentes únicamente de las explotaciones de sus socios a quienes garantiza todos los servicios necesarios para que desarrollen su actividad. Su creación se remonta a 1968, con la participación de 400 personas. Jesús García Calvo, elegido primer presidente de la cooperativa, fue el gran impulsor de la creación de esta cooperativa. En 1970 se construyó la primera fábrica de piensos de FEIRACO, que al año siguiente empezó a comercializar productos lácteos. En 1980 amplía sus servicios, ofreciendo a sus socios gasolineras, taller y veterinaria. La Cooperativa es también la entidad dominante del Grupo FEIRACO, que incluye FEIRACO Lácteos, S.L y FEIRACO Piensos, S.L.U. En el año 1995 se constituyó la Fundación Feiraco, cuyo objetivo fundacional es la adminsitración, gestión y aplicación del Fondo de Educación y Formación.

Desde sus inicios, FEIRACO ha mantenido una apuesta continua por la calidad, la innovación y la responsabilidad social. Así, el lanzamiento de la marca de leche premium Unicla le confirió distintos 
reconocimientos, entre los que destaca el Primer Premio Europeo a la Innovación Corporativa en 2009. En este mismo año se produce la adhesión de la corporación a la Red del Pacto Mundial de Naciones Unidas, como prueba de su compromiso por mantenerse actualizada en todo lo relacionado con el cumplimiento de los principios que marca la Responsabilidad Social Corporativa implantada por la Unión Europea y ratificada por España. En 2016 FEIRACO recibe el Premio Responsabilidad Social en Movimiento, concedido por la Xunta de Galicia.

En el año 2015, último año en el constan cuentas depositadas en el momento de elaboración de este trabajo, certificaba un total de 3.654 socios y socias de las cuales 3.378 son personas físicas. Su consejo rector se compone de once miembros, de los cuales tres son mujeres, siendo el presidente D. José Severino Montes Pérez.

OS IRMANDIÑOS, SOCIEDADE COOPERATIVA GALEGA. Os Irmandiños, Sociedade Cooperativa Galega (anteriormente denominada Cooperativa do Campo Agrícola - Gandeira e de elaboración de Penso Composto), se constituye el 6 de marzo de 1976 cuando 70 ganaderos de los ayuntamientos de Ribadeo, Barreiros, Trabajada, Lourenzá y Foz deciden construir una fábrica de pienso para abastecer sus explotaciones, principalmente de vacuno de leche. Oficialmente comienzan su actividad dos años después, y comienzan a asociarse en gran número más ganaderos, llegando a la actualidad con más de mil socios y con implantación en cerca de 40 municipios de Lugo y Asturias.

Durante el periodo de expansión no solo creció el número de socios de la cooperativa, sino que se pusieron en marcha múltiples actividades y servicios con el objetivo de cubrir todas las necesidades de las explotaciones realizadas por sus socios. Así, por ejemplo, en 1992 inicia su funcionamiento el parque de maquinaria. Este servicio pone al alcance de las explotaciones maquinaria de alto rendimiento y puntera en prestaciones que permite la calidad de los forrajes propios como activo importante dentro de la producción de leche y reducir los costes.

En los estatutos de la cooperativa se recogen, sin carácter de exclusividad, diferentes actividades:

- Elaboración de piensos compuestos para las explotaciones de los socios.

- La adquisición, por cualquier título, de animales, materias primas, instrumentos, maquinaria, fertilizantes, plantas, semillas, pesticidas, piensos compuestos; así como la de instalaciones relacionadas con la agricultura, la ganadería y de los bosques, tales como molinos, bodegas, fábricas de transformación, conservación y elaboración de productos.

- La prestación de servicios técnicos relacionados con la actividad económica, formativa y de apoyo a los socios.

- Cualquier otra actividad que sea necesaria, conveniente o que facilite la mejora económica, técnica, laboral o ecológica de la cooperativa o de las explotaciones de los socios.

- La cooperativa podrá, con carácter accesorio y subordinado, procurar bienes y servicios para el consumo de sus socios y de sus familiares que con ellos convivan. 
En el año 2016, previo al nacimiento de CLUN, certificaba en las cuentas anuales depositadas en el registro de cooperativas un total de 1.077 socios y socias de las cuales 842 son personas físicas (396 hombre y 446 mujeres). Su consejo rector se compone de ocho miembros, siendo el presidente D. José Ángel Blanco Purriños.

MELISANTO SOCIEDADE COOPERATIVA GALEGA- Localizada en Melide, esta cooperativa nace en 1979 de la mano de un grupo de agricultores y ganaderos de los municipios de Melide, Santiso e Toques. En 1989 añaden a sus actividades el servicio de atención veterinaria en las explotaciones de la agrupación, con la consiguiente contratación de un técnico veterinario orientado sobre todo a la realización de clínica por ser el servicio más solicitado por sus socios.

En 1990 amplían sus terrenos con la compra de 3.000 metros cuadrados de terreno para hacer frente a nuevas actividades, e inician el reparto de mercancía a domicilio a los socios con la contratación de personal ajeno en servicio. En esta década la cooperativa incluía las secciones de fábrica de piensos, almacén de distribución, tienda agraria, servicios veterinarios, economato, taller mecánico y cerrajería, ordeño y agrupación de productores de leche, gestión de explotaciones y servicios centrales. Todas estas secciones se fueron reorganizando en años sucesivos a petición de los socios, buscando soluciones a la problemática planteada en cada momento. En la actualidad cuenta con secciones de comercialización de leche cruda de vaca, carne de vacuno y productos hortícolas; fábrica de piensos; taller; economato; veterinario; maquinaria en común; alimentación con carro mezclador y gasóleo a domicilio.

Según consta en los estatutos actuales, el objeto social de la cooperativa es el abastecimiento, comercialización y elaboración de productos agrarios para las explotaciones de los socios y las siguientes actividades:

a) La comercialización con las transformaciones a que hubiera lugar de las producciones procedentes de las explotaciones agrícolas, ganaderas o forestales de las personas socias.

b) Con carácter específico se determina la leche y la carne de ganado vacuno.

c) La adquisición de materias primas, abonos, semillas y otros productos que puedan ser necesarios o convenientes para las explotaciones de los socios.

d) Adquirir, parcelar, sanear y mejorar terrenos destinados a la agricultura, la ganadería o los bosques, así como la construcción y explotación de las obras e instalaciones necesarias para estos fines.

e) Adquirir, elaborar, producir y fabricar, por cualquier procedimiento, para la Cooperativa o para las explotaciones de los socios, animales, piensos, abonos, plantas, semillas, insecticidas, instrumentos, maquinaria, instalaciones y cualesquiera otros elementos necesarios o convenientes para la producción y fomento agrario.

f) La prestación de servicios técnicos, formativos y de apoyo en general que puedan ser necesarios. 
g) Cualesquiera otras actividades que sean necesarias o se consideren convenientes para facilitar el mejoramiento económico, técnico, laboral o ecológico de la Cooperativa o de las explotaciones de sus asociados.

En el año 2015, último año en el constan cuentas depositadas en el momento de elaboración de este trabajo, certificaba un total de 697 socios y socias de las cuales 666 son personas físicas siendo 424 hombres y 242 mujeres. Su consejo rector se compone de 10 miembros, siendo el presidente D. Pablo Costoya Varela.

\section{b. Análisis económico}

El estudio económico de las entidades se realiza sobre las cuentas depositadas correspondientes al año 2015, solicitadas a los correspondientes registros de cooperativas. Se eligió este ejercicio por dos razones: ser el que se tomó como referencia para la realización del proyecto de integración; y no tener disponibles las cuentas correspondientes al ejercicio 2016, previo al nacimiento de CLUN.

Las tres entidades sometieron sus cuentas a una auditoría externa con entidades diferentes. Los informes fueron positivos en los tres casos, lo que sugiere que las tres cooperativas auditadas poseen unas cuentas que expresan, en todos los aspectos significativos, la imagen fiel de su patrimonio y de la situación financiera. Únicamente se consigna una salvedad en el informe correspondiente a Os Irmandiños, ligada a la difícil situación que atraviesa "Atesáns Gandeiros, S.C.G", participada por la entidad.

Las entidades arrojan resultados positivos al cierre del ejercicio de referencia:

\section{Tabla 3. Excedentes netos de cada una de las cooperativas. Año 2015}

Cooperativa

Excedentes del ejercicio 2015

FEIRACO

$110.401 €$

OS IRMANDIÑOS

$56.682 €$

MELISANTO

$107.081 €$

FUENTE: Elaboración propia a partir de las cuentas de las entidades analizadas. 
Como se observa en la tabla 3, la entidad con menor excedente es Os Irmandiños. De hecho, se registra un descenso del $86 \%$ en relación con el ejercicio precedente, debido a las dificultades de cobro de la empresa participada anteriormente destacada (Atesáns Gandeiros, SCG). En lo referente a la actuación con el excedente las tres obran de formas diferentes: Feiraco dedica el $100 \%$ de sus excedentes a compensar pérdidas de ejercicios anteriores; Os Irmandiños dota los fondos obligatorios (formación y reserva) con las cantidades mínimas que indica la ley (5 y 20\% respectivamente) y el $75 \%$ restante decide dotarlo a un fondo de reserva voluntario. Melisanto dota igualmente los fondos obligatorios con las mínimas cantidades, y el excedente restante lo dota a un fondo de reserva voluntaria irrepartible. Por tanto, aun bajo fórmulas diferentes, las tres entidades optan por reforzar las cooperativas ayudando a su capitalización.

\section{Tabla 4. Cifras de negocio del año 2015}

\begin{tabular}{|l|r|r|r|}
\hline División & Feiraco & Os Irmandiños & Melisanto \\
\hline Lácteos & $45.302 .219 €$ & $14.196 .242 €$ & $21.718 .534 €$ \\
Piensos & $20.193 .256 €$ & & \\
Mercado rural, economato, abonos & $8.581 .790 €$ & $5.921 .303 €$ & \\
Cogeneración & $2.421 .646 €$ & & \\
Petróleo, taller y otros & $6.585 .411 €$ & & \\
Servicios & $229.864 €$ & $336.810 €$ & \\
Alimentación complementaria & & $757.318 €$ & \\
Abonos & & $1.137 .882 €$ & \\
Venta de terneros & & $31.495 €$ & $-8.077 €$ \\
Mezcla Húmeda & & $1.721 .090 €$ & $-113.173 €$ \\
Servicios de maquinaria & & $-8.488 €$ & $-3.407 .907 €$ \\
Rappels & & & $18.787 .608 €$ \\
Descuentos & & & \\
Devoluciones & & $32.643 .319 €$ & \\
\hline TOTAL & & & \\
\hline
\end{tabular}

FUENTE: Elaboración propia a partir de las cuentas de las entidades analizadas.

El análisis de las cifras de negocio (Tabla 4) sugiere alguna cuestión relevante. En primer lugar, se puede observar el claro predominio en las cifras de negocio de FEIRACO. De hecho, el conjunto de las otras entidades participantes no alcanza el $62 \%$ de la cifra registrada por esta cooperativa. En 
segundo lugar, se constata la complementariedad del objeto de negocio de las tres organizaciones, con áreas más complementarias que comunes en su objeto de negocio. Así, la única partida común es la de "lácteos", además de la genérica de "servicios" que hace referencia a una cartera no necesariamente común. A este respecto, cabe destacar que esta complementariedad en el marco del modelo cooperativo ha sido considerada un mecanismo óptimo para acercar el sector primario al mercado final, integrando en la misma entidad la gestión de la producción, transformación y comercialización agroalimentaria (Meliá y Peris, 2017). Finalmente, se observa también la menor diversificación de Melisanto en relación con las otras dos cooperativas.

La tabla 5 permite observar los costes de personal de las tres entidades, así como el coste medio por persona en el ejercicio 2015. Se puede comprobar que tanto los costes como el número de personas contratadas responden al orden anteriormente establecido en cuanto a cifra de negocios. No obstante, las diferencias son menores que las correspondientes a dicha cifra. Destaca particularmente la homogeneidad del coste medio por persona, con un intervalo de variación inferior a 800,00 euros entre las tres sociedades. Asimismo, si ponemos en relación las cifras del coste de personal con las correspondientes a la cifra de negocios podemos intuir que el modelo de negocio en cada una de las cooperativas es diferente 0 se desarrolla de forma muy diferenciada.

\section{Tabla 5. Costes de personal, 2015}

\begin{tabular}{|l|c|c|c|}
\hline Cooperativa & Coste de personal & Personal 2015 & Coste medio por persona \\
\hline FEIRACO & $3.158 .454 €$ & 108 & $29.245 €$ \\
OS IRMANDIÑOS & $2.863 .876 €$ & 99 & $28.928 €$ \\
MELISANTO & $1.187 .874 €$ & 40 & $29.697 €$ \\
\hline
\end{tabular}

FUENTE: Elaboración propia a partir de las cuentas de las entidades analizadas.

Avanzamos ahora en el análisis económico-financiero de las distintas entidades. Para ello, examinamos en primer lugar la composición de los distintos balances de situación (Tabla 6). En este sentido, cabe destacar que la aplicación de la normativa contable determina que la totalidad del capital social figura en el pasivo corriente de las cooperativas, a diferencia del resto de formas societarias. 


\section{Tabla 6. Porcentaje de masas patrimoniales sobre la estructura financiera (2015)}

\begin{tabular}{|l|c|c|c|c|c|}
\hline \multirow{2}{*}{ Cooperativa } & \multicolumn{2}{|c|}{ ACTIVO } & \multicolumn{3}{c|}{$\begin{array}{c}\text { PASIVO } \\
\text { Corriente }\end{array}$} \\
& Corriente & No corriente & Patrimonio Neto & \\
\hline FEIRACO & $48,44 \%$ & $51,56 \%$ & $8,87 \%$ & $55,87 \%$ & $35,25 \%$ \\
OS IRMANDIÑNOS & $45,25 \%$ & $54,75 \%$ & $36,67 \%$ & $36,29 \%$ & $27,04 \%$ \\
MELISANTO & $37,12 \%$ & $62,88 \%$ & $36,99 \%$ & $35,84 \%$ & $27,16 \%$ \\
\hline
\end{tabular}

FUENTE: Elaboración propia a partir de las cuentas de las entidades analizadas.

Esta información se completa con la de la Tabla 7, donde vemos las principales cifras incluidas en el balance de situación de cada sociedad a finales de 2015.

\section{Tabla 7. Activo y Pasivo de las diferentes cooperativas, año 2015}

\begin{tabular}{|l|r|r|r|r|r|}
\hline \multirow{2}{*}{ Cooperativa } & \multicolumn{2}{|c|}{ ACTIVO } & \multicolumn{3}{c|}{ PASIVO } \\
\hline FEIRACO & \multicolumn{1}{|c|}{ Corriente } & No corriente & Patrimonio Neto & \multicolumn{1}{c|}{ Corriente } & No Corriente \\
OS IRMANDINOS & $13.750 .855 €$ & $14.636 .219 €$ & $2.519 .102 €$ & $15.861 .119 €$ & $10.006 .853 €$ \\
MELISANTO & $8.903 .100 €$ & $10.771 .994 €$ & $7.214 .809 €$ & $71.403 .280 €$ & $5.319 .957 €$ \\
& $2.656 .159 €$ & $4.498 .666 €$ & $2.646 .705 €$ & $2.564 .620 €$ & $1.943 .500 €$ \\
\hline
\end{tabular}

FUENTE: Elaboración propia a partir de las cuentas de las entidades analizadas.

Se trata de tres cooperativas de tamaño medio-pequeño, con una posición más dominante de FElRACO. A este respecto, podemos señalar que el estudio de Meliá-Martí y otros (2015) encuentra que la menor dimensión inicial agregada de las cooperativas aumenta la posibilidad de éxito del proceso de integración en casos de fusión. En este mismo estudio se analiza la hipótesis (no demostrada) que las diferencias de tamaño entre las cooperativas que se fusionan no influyen de forma significativa en la probabilidad de éxito. Asimismo, otro elemento determinante del éxito en el caso de las fusiones es el indicador que hace referencia al dimensionamiento de las organizaciones, concretamente el volumen de activos sobre el valor de la producción agregada inicial. Por tanto, los datos sugieren cierto optimismo en la probabilidad de éxito en la integración. 
Desde el nacimiento de CLUN cada una de las cooperativas partícipes procederá a aportar la totalidad de sus negocios -activos y personal- a la nueva cooperativa la cual, a partir de la integración, pasará a realizar todas las actividades frente a terceros. En cuanto al número de entidades el nuevo grupo contará con participaciones significativas en las sociedades Feiraco Piensos ${ }^{14}$, Feiraco Lácteos, Clesa-Acolat ${ }^{15}$, Ucoga Asesores, Delagro ${ }^{16}$ y Artesans Gandeiros ${ }^{17}$.

\section{c. Determinación de la participación en CLUN}

Como se dijo previamente, las cooperativas de segundo grado tienen como objeto la promoción, coordinación o desarrollo en común de determinadas actividades en beneficio de sus socios (Juliá Igual et alt., 2004). En el caso de CLUN este principio se traduce fundamentalmente en la transformación y venta de productos lácteos y la elaboración de forraje y piensos.

Debe señalarse que las tres cooperativas tenían experiencia previa en la colaboración, puesto que habían cooperado juntas en otros negocios, principalmente en el sector lácteo con CLESA, conocida desde 2012 como ACOLAT (Agrupación de Cooperativas Lácteas, S.L) ${ }^{18}$.

Otro punto favorable es la complementariedad de las estructuras que cada una aportaba a la nueva entidad. Así, si la fortaleza de Feiraco se encuentra en la transformación y distribución de productos lácteos -con acuerdos de venta con los principales distribuidores de alimentación-, Melisanto y Os Irmandiños destacan en la producción de piensos y forraje para alimentación, además de contar con numerosa maquinaria para su recogida y elaboración. Con la cooperativa de segundo grado se procede a la eliminación de duplicidades entre las tres estructuras. Por tanto, el establecimiento de una cadena de valor complementaria depurada de duplicidades ${ }^{19}$ permite abarcar más servicios a los socios y clientes que anteriormente.

14.- Proviene, junto con Feiraco Lácteos y Ucoga Asesores, de Feiraco.

15.- Proviene de Feiraco, Os Irmandiños y Melisanto. Con la marca comercial Clesa, Agrupación de Cooperativas Lácteas SL - ACOLAT está conformada por 11 cooperativas gallegas, que trabajan conjuntamente a través para realizar derivados lácteos.

16.- Proviene de Feiraco, Os Irmandiños y Melisanto. Es una cooperativa de segundo grado, integrada, a su vez, por más de 26 cooperativas y entidades, algunas de ellas, de segundo grado de Asturias, Cantabria y Galicia que, juntas aportan más de 46 cooperativas representadas. Entre los servicios a los que los socios tienen acceso, se encuentran la gestión profesional de compras de suministros agrarios, atención, información, formación y asesoramiento a las cooperativas asociadas. Cuenta con diversos supermercados y tiendas agrarias, bajo la insignia Mercarural, tanto en Asturias como en Cantabria y en Galicia.

17.- Proviene de Os Irmandiños, es una Cooperativa de segundo grado que integra diez cooperativas socias, con más de 4000 productores. Ofrece servicios de comercialización de ganado de vacas e asesoramiento técnico.

18.- En 2011 la crisis concocida como "nueva Rumasa" afectaba de lleno a CLESA, entidad con más de 70 años de trayectoria en el sector lácteo en Galicia con origen en Lácteas del Atlántico (filial del Grupo Centrales Lecheras Españolas S.A.). En el momento de la liquidación concursal un grupo de cooperativas, entre las que se encuentran Feiraco, Os Irmandiños y Melisanto, deciden hacerse con la propiedad de la factoría como estrategia de transformación y venta de su materia prima. El proyecto arranca en el 2012 con una producción de 3.500 toneladas de yogur que en el 2016 llegarían a las 30.000, siempre con una estrategia de valorizar los lácteos, subir los precios al ganadero, y que el mercado garantice la sostenibilidad de toda la cadena.

19.- Por ejemplo, se reestructura la sección de "recursos lecheros" ampliando los servicios, reubicando al personal y estableciendo claras secciones $y$ actividades. 


\section{CLUN: CONCENTRACIÓN POR FUSIÓN DE TRES COOPERATIVAS LÁCTEAS EN EL COOPERATIVISMO AGRARIO EN GALICIA}

Podemos determinar por tanto que la concentración se lleva a cabo para aportar dos tipos de soluciones: internas y externas. Las primeras versan sobre la prestación de servicios y de suministros a las cooperativas socias. Las segundas están pensadas para mejorar los procesos de fabricación y transformación de la materia prima.

Para llevar a cabo el proceso de integración, las cooperativas implicadas en el proceso designaron un experto externo, AT Consultores \& Auditores, S.L.U, quien, sobre la base de los balances de las cooperativas a 31 de diciembre de 2015 , realizó una valoración de cada una de ellas a efectos de determinar el porcentaje de participación en el capital de la nueva entidad. La valoración realizada por la consultora a cada una de las tres cooperativas se hizo de acuerdo con métodos de valoración generalmente aceptados, y que consisten en considerar, para cada una de las cooperativas, su expectativa de generación de caja en el futuro, deduciendo su deuda financiera en el momento de la valoración. La metodología de valoración fue homogénea para las tres cooperativas.

De esta forma se determinaron el valor y los porcentajes de participación en el capital de CLUN por AT Consultores resultando de la siguiente manera:

\section{Tabla 8. Resultados de la valoración y porcentajes de participación en CLUN}

\begin{tabular}{|c|c|c|}
\hline Cooperativa & IMPORTE & PORCENTAJE \\
\hline MELISANTO & $4.760 .690 €$ & $14,59 \%$ \\
\hline OS IRMANDIÑOS & $11.559 .484 €$ & $35,41 \%$ \\
\hline FEIRACO & $16.320 .174 €$ & $50,00 \%$ \\
\hline TOTAL & $32.640 .348 €$ & $100,00 \%$ \\
\hline
\end{tabular}

FUENTE: Elaboración propia con datos de AT Consultores.

Como podemos observar la participación no es igualitaria entre las tres entidades socias. La que obtiene un mayor porcentaje es Feiraco, con la mitad de la participación; seguida de Os Irmandiños e Melisanto. 


\section{d. Análisis organizativo}

El 31 de octubre de 2016, las Asambleas Generales extraordinarias de socios de Feiraco, Melisanto y Os Irmandiños, aprobaron unirse en una Cooperativa de segundo grado de integración empresarial, de nueva creación, con el objetivo de crear un grupo cooperativo de referencia en el sector agro ganadero, que permitiese ganar tamaño y eficiencia a corto plazo y, así, convertirse en un grupo líder en la economía de la cornisa cantábrica. La integración se hizo pública el 01/01/2017. Juntas, las tres cooperativas gallegas buscan mejorar la comercialización de los productos lácteos y crecer con las marcas que ya poseen: Feiraco, Arquega, Clesa y Unicla.

CLUN representa el conjunto de 3.559 socios y 406 trabajadores, que producen el $15 \%$ de la leche gallega y transforman 152 millones de litros anuales a través de las marcas Feiraco, Unicla y Clesa. También se comercializa leche de almendras bajo la marca Almond Breeze y queso a través de la quesería Arquega.

El modelo de organización cooperativo permite controlar y optimiza todas las etapas del proceso productivo gestionando con un sistema integrado de calidad y de inocuidad de los alimentos. Para llevar a cabo estas labores, uno de los mayores retos a los que se enfrenta la nueva organización en los primeros años de su existencia es, sin duda, el organizativo, ya que tienen que tienen que formar y consolidar los equipos directivos resultantes de la integración al tiempo que profundiza en la definición de las áreas de negocio y se da respuesta a las demandas de clientes y explotaciones socias de las cooperativas de primer grado.

Como ya se ha referido en reiteradas ocasiones, el caso que nos ocupa es una cooperativa de $2^{\circ}$ grado y por lo tanto las cooperativas que se integran siguen funcionando, transfiriendo los activos necesarios a la entidad resultante. Es decir, nos encontramos con tres cooperativas que siguen con su funcionamiento, pero que centralizan sus actividades principales en CLUN, siendo además esta la que soporta la mayor parte de la facturación al ser la encargada de las ventas tanto de piensos y forrajes como de leche y sus derivados.

En la estructura organizativa de CLUN se identifican tres divisiones 20 :

- Lácteos, encargada de la recogida, envasado y distribución de leche y otros derivados y se encuentra ubicada en ACOLAT. La cooperativa comercializa productos lácteos bajo las marcas de Feiraco, leche Premium Únicla, yogures y postres lácteos Clesa y quesos Arquega.

- Suministros, encargada de proporcionar al socio los productos necesarios para el desarrollo de su actividad, con fábricas de producción de piensos, surtidores de gasóleo y tiendas agrarias y con ubicación principal en Ribadeo, sede de Os Irmandiños.

20.- Anteriormente Feiraco SCG poseía tres negocios: Feiraco Lácteos, Feiraco Piensos y Sociedad Cooperativa, sobre la base de la simplicidad en el reparto de la cuenta de resultados y la división de negocios que favorecía la organización de equipos de trabajadores. Actualmente estas áreas siguen vigentes como subdivisiones, a pesar de la integración. 
- Servicios, emplazada en Feiraco. Esta sección permite proveer al socio de todo cuanto pueda necesitar para la mejora de la gestión de su ganadería, desde apoyo económico y financiero o técnico hasta servicios de gestión administrativa, aseguramiento, compercialización, maquinaria 0 reparaciones.

Existen también tres áreas transversales que prestan servicios a toda la estructura de CLUN: el Departamento de Recursos Humanos, IT y Finanzas (contabilidad) ${ }^{21}$. Como podemos observar, la nueva estructura organizativa sienta sus pilares en la unión cooperativa y la economía social, por lo que se organiza en seis direcciones: tres de negocio (campo y explotaciones, alimentación animal y láctos) y tres transversales (personas-responsabilidad social, recursos económicos y sistemas de información).

Esta forma de funcionamiento permite a las cooperativas de primer grado mantener las divisiones de negocio que poseían previas a la integración pero con una coordinación global desde la cooperativa resultante, hechos que deberían ayudar a que cada uno de los socios alcance sus objetivos (Arcas Lario, Munuera Alemán, y Hernández Espallardo, 2002). Tanto Feiraco como Os Irmandiños y Melisanto continúan teniendo su domicilio social (Ames - Pontemaceira, Ribadeo y Melide, respectivamente) y realizan las mismas actividades principales e incluso mantienen sus secciones (sección láctea, piensos, veterinaria, etc.) pero la facturación consta a nombre de CLUN.

En lo referente a los empleos, como cada actividad sigue prestándose donde venía realizándose, no existieron flujos de trabajadores de unas cooperativas a otras o de unas localidades a otras. De hecho, dentro del organigrama, los distintos tipos de dirección se reparten según las fortalezas de cada cooperativa: por ejemplo, en Ribadeo (Os Irmandiños) se encuentra ubicada la dirección de servicios al socio; en Acolat ("Clesa", Caldas de Reis) se encuentra el Director de la División Láctea. En la actualidad se está trabajando en la negociación de un convenio colectivo aplicable a CLUN y a sus socias, con el objetivo primordial de que todos los trabajadores, aunque no pertenezcan a la misma actividad o naturaleza de actividad, se rijan por los mismos derechos unificando de esta manera la normativa laboral a aplicar.

CLUN se sitúa en una treintena de municipios gallegos del centro y norte de Galicia, considerados zonas estratégicas para el desarrollo del sector lácteo. La propia organización reconoce que la homogeneización territorial permite ganar presencia; volumen de negocio; unificar procesos y técnicas; vertebrar la cadena de valor con criterios compartidos en contextos globales; obtener beneficios para todos los estamentos; ser más competitivos, eficaces y eficientes para poner en valor los recursos naturales de Galicia. CLUN cuenta con un cuadro de personal de 406 trabajadores en empleos directos, además de los indirectos asociados a sus actividades.

21.- En el acuerdo de integración se determina que Feiraco será la principal gestora de CLUN, por lo que estas áreas transversales se ubican en la sede que tiene esta cooperativa en Pontemaceira (Negreira). No existe ninguna razón de aportación económica o posesión de poder mayoritario, simplemente se elige por su ubicación y por la dimensión de sus instalaciones. 
En cuanto a la organización societaria, el Consejo Rector de la nueva entidad está formado por los tres presidentes de las cooperativas socias, cada uno con un cargo. Esta representación garantiza la participación de las tres cooperativas en la gestión. Así, figura como presidente el representante de Os Irmandiños, como vicepresidente el representante de Feiraco y como secretario el de Melisanto. Estas personas mantienen los cargos ocupados anteriormente en sus correspondientes cooperativas.

Finalmente, en este primer año CLUN ha alcanzado facturación de 125 millones de euros, a los que hay que añadir 76 millones de Feiraco Lácteos y 27 millones de ACOLAT-CLESA. La cooperativa produjo 200.000 toneladas de alimentación animal, y la transformación superó los 160 millones de litros de leche. A título comparativo, FEIRACO procesó en 2014126 millones de litros de leche y produjo 68.000 toneladas de alimentación de alimentación, facturando un total de 118 millones de euros.

La cooperativa ha llevado a cabo una inversión, prevista en su plan económico, de más de 5,5 millones de euros en una nueva línea de envasado para el nuevo formato de cartones de leche de las marcas Feiraco y Unicla. En este sentido, ha sido la primera láctea con envases con certificación "carbón natural", dejando de emitir cerca de 1.650T de dióxido de carbono. Además, ha invertido en la adquisición de nueva maquinaria en vistas a la renovación de la utilizada por los Irmandiños para prestar servicios agrícolas o de alimentación; y en tecnologías de la información (para avanzar en la centralización informática). En el marco de estas inversiones ha reforzado una apuesta importante en I+D+i22, participando en distintos proyectos entre los que destaca Cattlecare, en colaboración con la empresa Gradiant, que pretende mejorar la monitorización de las reses para extraer datos del comportamiento del ganado y establecer patrones de comportamiento que permitan detectar anomalías 0 que sean indicadores de enfermedades, periodo de celo o procesos de parto. Además, trabaja en la mejora de la gestión de los recursos en las explotaciones, mediante la automatización y la optimización de la logística de distribución.

\section{e. Retos de futuro}

La cooperativa de segundo grado pone su foco en cuatro grandes retos: la eficacia, la calidad, innovación y la internacionalización. Estos son los ejes sobre los que se debe asentar la producción láctea y que en palabras del director general de CLUN, José Luis Antuña, solo se le puede dar respuesta con la "unión cooperativa y la economía social".

22.- Cabe destacar que esta apuesta por la innovación es consustancial a Feiraco. Por ejemplo, en el período 2013-2014 la inversión en innovación superó 1,4 millones de euros en programas como Tecopaga, para el diagnóstico y mejora del status sanitario de los animales; $Q$ Safe, herramienta cuantitativa para alimentos y energía sostenibles en la cadena alimentaria; NewGalimentos, para el desarrollo de nuevos alimentos con calidad diferenciada; Clean-Label para el estudio de alternativas para eliminación de aditivos o Susmilk, programa de optimización del consumo de energía y agua y aprovechamiento de recursos energéticos renovables. 
Desde su constitución en enero de 2017, Cooperativas Lácteas Unidas ha conseguido avanzar en la consecución de sus objetivos estratégicos: potenciar los servicios técnico-económicos de la cooperativa para una mayor eficiencia de los costes de producción de sus socios; optimización del precio de los suministros en mejores condiciones de competitividad; y ganar volumen para mejorar la cadena de valor del agro gallego y contribuir a su estructuración, impulsando la sostenibilidad de la producción láctea ${ }^{23}$. A pesar de que tan solo lleva en funcionamiento poco más de un año, esta cooperativa ya ha obtenido algún reconocimiento. Así es que el 30 de mayo de 2018, CLUN recibe el premio a la mejor cooperativa del año, otorgado por Cooperativas Agroalimentarias de España, una iniciativa de carácter anual que tiene por objetivo el reconocimiento de la labor y el esfuerzo de las cooperativas en ámbitos concretos en tres categorías: Desarrollo Rural, Igualdad de oportunidades e Innovación 24 .

Una de las preocupaciones inmediatas de CLUN, con incidencia directa en las posibilidades de consecución de estos retos, es el avance tecnológico. En consonancia, se centran en la búsqueda de fórmulas de producción con las que poder producir más rápido y eficientemente, respetando siempre las medidas de impacto medioambiental que establece la UE.

Pero la dirección de CLUN de Cooperativas Lácteas Unidas se propone unos objetivos clave para continuar y mejorar su repercusión y éxito en el mercado del sector lácteo, a nivel gallego, español y europeo.

Estos son:

- Costes: Integración de los servicios técnicos financieros para alcanzar una mayor eficiencia de los costes de producción. En un contexto global en el que el sector lácteo está condicionado por la fuerte volatilidad de los precios que afecta a las explotaciones socias tanto por el tamaño reducido de sus empresas como por la falta de integración en un proceso industrial que aporte retorno cooperativo.

- Calidad: Optimizar los suministros en las mejores condiciones competitivas, dado el efecto directo que las materias primas de alimentación animal y otros suministros representan en la competitividad de las granjas. En este contexto, la leche Únicla ha obtenido la certificación del análisis del ciclo de vida del proceso productivo de la leche de AENOR, siendo uno de los escasos ejemplos que existen de análisis del ciclo de vida en el sector alimentario "desde la cuna hasta la puerta" al incluir desde el cambio del uso de la tierra para el cultivo de alimentos para el ganado, forrajes, piensos, maquinaria agrícola, vacas de todas las edades, instalaciones ganaderas, transportes, envasado en la fábrica y transporte hasta la distribución. Asimismo, es una de las primeras

23.- Asociación Galega de Cooperativas Agroalimentarias, AGACA (2018). Nota de prensa: CLUN, premio "Cooperativa del Año" de Cooperativas Agro-alimentarias de España, http://agaca.coop/clun-premio-cooperativa-del-ano-de-cooperativas-agro-alimentarias-de-espana/.

24. - La entidad ha reconocido a CLUN con este galardón como "una de las apuestas de integración cooperativa más importantes de los últimos años" y por "haberse convertido en referente en el sector agro-ganadero gallego y su compromiso con la responsabilidad social, la innovación, la calidad y su modelo de gestión orientado a una sostenibilidad económica, social y medioambiental en el que las personas son lo primero". 
organizaciones que ha obtenido la certificación de Bienestar Animal y de seguridad alimentaria ISO 22000.

- Trabajo: CLUN permite agregar volumen para mejorar la cadena de valor y contribuir a su estructuración. Todo ello contribuye a la sostenibilidad del sector primario.

De forma inmediata, CLUN debe centrarse en conseguir retornos cooperativos que satisfagan a todos sus socios; continuar los esfuerzos dedicados a la implementación de procesos y modelos de costes de eficiencia y producción que mejoren las condiciones de todos los socios. Asimismo, debe contribuir a la recuperación del valor de la leche y su consumo. Al no existir productos sustitutivos a la leche líquida, cuyo consumo cae constantemente, el foco de la cooperativa es el comercio con los países emergentes, grandes demandantes de lácteos. En este apartado, el sureste asiático y el norte de África se consolidan como mercados exteriores preferentes, focalizando el eje de crecimiento de la compañía. A este respecto, trabaja en envases y formatos innovadores que permitan alargar la vida de los productos para superar la distancia geográfica.

Con todo, el reto más inmediato debe ser proseguir en la integración, con particular atención a la homogeneización de plantillas y equiparación de derechos y beneficios entre los trabajadores. La asimetría de convenios, heterogeneidad de funciones y carencia de un órgano de representación colectiva como mecanismo de negociación puede introducir disfunciones en el proceso de integración que, además, generarían amenazas de forma inmediata. Por tanto, debe figurar como prioridad en la agenda de trabajo. Otro de los retos que debe afrontar la entidad es la participación de las mujeres en los órganos de dirección. Sin duda esta circunstancia viene determinada por la escasa, en algún caso nula existencia de mujeres en los consejos rectores de las cooperativas de primer grado aun cuando el número de mujeres social es importante. Parece necesario estudiar las motivaciones que llevan a esta circunstancia para así identificar posibles techos de cristal o suelos pegajosos que impidan la normal promoción de las mujeres dentro de las organizaciones.

A largo plazo, CLUN no cierra puertas a la cooperación con otras industrias o fusiones con otras cooperativas agrarias. Destaca su presidente, José Ángel Blanco, que, para acercarse, por lo menos, a la competitividad que poseen las grandes cooperativas de Europa (la danesa Arla Foods o la francesa Sodiaal) no deben cerrar puertas a "alianzas que generen valor para la producción láctea y para nuestros socios ganaderos".

Externamente CLUN debe liderar, por su peso específico en el sector, junto la administración y el resto de los actores la elaboración de un plan, una estrategia para el sector lácteo gallego y del conjunto del estado como existen en los países de nuestro entorno como Francia, Italia o Reino Unido. 


\section{CLUN: CONCENTRACIÓN POR FUSIÓN DE TRES COOPERATIVAS LÁCTEAS EN EL COOPERATIVISMO AGRARIO EN GALICIA}

\section{Conclusiones}

Es pronto todavía para determinar el éxito o no del proceso de integración de CLUN, ya que viene de cerrarse el primer ejercicio económico y ni siquiera están las cuentas aprobadas. No obstante, de los factores que tradicionalmente se han relacionado con la competitividad de las empresas agroalimentarias (Lajara-Camilleri y Server-Izquierdo, 2017) en CLUN concurren al menos tres: tamaño (ArcasLario et al., 2014; Juliá et al., 2012); diversificación (Juliá et al., 2012; Campos-Climent y Chaves-Ávila, 2012) y orientación al mercado (Hernández-Espallardo y Arcas-Lario, 2003). En consecuencia, si se consigue culminar el acoplamiento organizativo, se sentarán las bases de una organización con características y capacidades importantes para competir con garantías en el mercado.

Además, toda vez analizado el proceso de concentración, podemos destacar una serie de buenas prácticas que favorecieron su realización. Algunas están ligadas a la propia idiosincrasia de las entidades, y otras surgieron a raíz de los procedimientos implantados en el transcurso de la integración.

En primer lugar, pese a que el objeto social de las entidades es coincidente (recogida y comercialización de la leche), la diferente especialización en servicios las convierte en organizaciones complementarias: Feiraco ocupa un rol protagonista en la comercialización y distribución; Os Irmandiños en la fabricación de piensos y Melisanto cuenta con una importante cartera de servicios para sus socios. Esta complementariedad ayudó, por ejemplo, a que no se realizasen movimientos de trasvase de personal y a facilitar la fijación de los centros de decisión de las diferentes divisiones.

En segundo término, nos gustaría destacar el importante conocimiento que las tres cooperativas tenían entre sí, ya que las tres venían compartiendo espacios en diferentes organizaciones en los últimos años. Como ya indicamos las tres entidades forman parte ACOLAT, dueña de la planta de derivados lácteos CLESA, y de la cooperativa de segundo grado Delagro. Además, las tres entidades forman parte de la Asociación de Cooperativas Agrarias de Galicia -AGACA- en cuyo consejo rector participaron Feiraco y Os Irmandiños, ostentando la presidencia y vicepresidencias respectivamente, durante el período que transcurre entre 2013 y 2017. Este conocimiento mutuo ayuda a que los procesos de fusión se realicen de forma natural reduciendo las posibles fricciones.

Esta circunstancia, junto a que todas las entidades afectadas por el proceso de integración sean cooperativas y se rijan por los mismos principios, parece indicar que tengan culturas empresariales semejantes. Para el éxito de cualquier proceso de concentración, además del ajuste financiero, empresarial y organizativo, es imprescindible el alineamiento social. Por tanto, la combinación de una cultura empresarial similar y equipos de gestión coordinados puede ayudar al buen fin del proceso. No obstante, volvemos a insistir en la necesidad de proseguir en el avance de la equiparación de derechos, obligaciones y beneficios entre los trabajadores, de cara a eliminar riesgos en la integración. 
Por último, también es destacable la elección conjunta y común de un mismo actor externo e independiente para la realización de la propuesta de valorización de cada organización y posterior determinación del porcentaje de participación de cada una de ellas en la entidad resultante. De esta forma se realiza de forma procedimental y ampliamente aceptada un proceso que, a priori, podría ser un foco de conflicto en el proceso de integración.

Finalmente, es relevante destacar la claridad de objetivo a largo plazo de la concentración. Esta nace con ambición y vocación de futuro, y el correcto acoplamiento de CLUN es un primer paso determinante para este horizonte de creación de un gran grupo lácteo gallego. En este camino, la recientemente creada Aira figura ya en el horizonte.

\section{Bibliografía}

ARCAS, N., MUNUERA, J. \& HERNÁNDEZ, M. (2002): "Beneficios de las cooperativas agrarias de segundo grado: contribución a los objetivos de sus socios", REVESCO, Revista de Estudios Cooperativos, 76, 7-25.

ARCAS-LARIO, N., MARTÍN-UGEDO, J.F. \& MÍNGUEZ-VERA, A. (2014): "Farmers' Satisfaction with Fresh Fruit and Vegetable Marketing Spanish Co-operatives: An Explanation from Agency Theory", International Food and Agribusiness Management Review, 17(1), 127-146.

BABÍO, M.R. \& JORDÁN, M. (2008): Cooperativas de Galicia. Informe de Síntese 2007, Vol. 1, Xunta de Galicia.

BABÍO, M.R. \& JORDÁN, M. (2010): Cooperativas de Galicia. Informe de Síntesis 2010, Xunta de Galicia.

BARREIRO, M.J. (2013): As raices históricas do Cooperativismo agrario en Galicia (1890-1936), Edita: CECOOP, Centro de Estudios Cooperativos, Universidad de Santiago de Compostela.

BRETOS, I., DÍAZ-FONCEA, M. \& MARCUELLO, C. (2018): "Cooperativas e internacionalización: Un análisis de las 300 mayores cooperativas del mundo", CIRIEC-España, Revista de Economía Pública, Social y Cooperativa, 92, 5-37. DOI: 10.7203/CIRIEC-E.92.11480.

CAMPOS-CLIMENT, V. \& CHAVES-ÁVILA, R. (2012): "El papel de las cooperativas en la crisis agraria. Estudio empírico aplicado a la agricultura mediterránea española", Cuadernos de desarrollo rural, 9 (69), 175-194.

CHAVES, R. (1996): "La economía Social ante los desafíos de la cooperación y la concentración empresarial". En: CIRIEC-España (Ed.), Informe sobre la situación de las cooperativas y las sociedades laborales en España, 314-370. 
COOPERATIVAS AGRO-ALIMENTARIAS DE ESPAÑA (2018): El cooperativismo agro-alimentario. Macromagnitudes del Cooperativismo Agrario Español, Observatorio Socioeconómico del Cooperativismo Agroalimentario Español (OSCAE).

FAYOS, T., CALDERÓN, H. \& MIR, J. (2011): "El éxito en la internacionalización de las cooperativas agroalimentarias españolas. Propuesta de un modelo de estudio desde la perspectiva del marketing internacional", CIRIEC-España, Revista de Economía Pública, Social y Cooperativa, 72, 43-72.

FLECHA, R. \& NGAI, P. (2014): "The challenge for Mondragon: Searching for the cooperative values in times of internationalization", Organization, 21(5), 666-682.

http://dx.doi.org/10.1177/1350508414537625.

GÓMEZ, N. (2015): 25 años de cooperativismo agroalimentario, Ed. Cajamar Caja Rural.

MINISTERIO DE AGRICULTURA, PESCA Y ALIMENTACIÓN (2018): Informe de coyuntura del sector vacuno de leche. Junio 2018.

MORALES, A.C., ROMERO \& MÚÑOZ, M.D. (2003): "Causas, intereses y desarrollo histórico de la acción colectiva empresarial en el ámbito agrario: el caso del cooperativismo en el sector oleícola", CIRIEC-España, Revista de Economía Pública, Social y Cooperativa, 46, 117-150.

HERNÁNDEZ-ESPALLARDO, M. \& ARCAS-LARIO, N. (2003): "The effects of authoritative mechanisms of coordination on market orientation in asymmetrical channel partnerships", International Journal of Research in Marketing, 20(2), 133-152. DOI: 10.1016/50167-8116 (03)00015-6.

JORDÁN, M., COUTINHO, M. \& MOUGÁN, H. (2004): Libro Branco do cooperativismo en Galicia, Xunta de Galicia.

JULIÁ, J.F., GARCÍA, G. \& MELIÁ, E. (2012): "La globalización y los modelos de crecimiento de los grupos cooperativos. Las cooperativas agroalimentarias en España y la Unión Europea", Ekonomiaz, Revista Vasca de Economía, 79, 83-113.

JULIÁ-IGUAL, J.F., MELIÁ-MARTÍ, E. \& GARCÍA-MARTÍNEZ, G. (2012): "Strategies developed by leading EU agrifood cooperatives in their growth models", Service Business, 6(1), 27-46. DOI: 10.1007/s11628-011-0129-3.

JULIÁ, J.F., SERVER, R.J. \& MELIÁ, E. (2004): Los procesos de fusión en cooperativas agrarias, Manual de procedimiento, Mundi Prensa Libros S.A. (1st ed.).

JULIÁ, J.F., MELIÁ, E., GARCÍA, G. \& GALLEGO, P.L. (2010): "Los factores de competitividad de las cooperativas líderes en el sector agroalimentario europeo. Acciones a emprender por las cooperativas agrarias españolas", Colección Económica, 14, Fundación Cajamar, Almeria.

LAJARA-CAMILLERI, N. \& SERVER-IZQUIERDO, R. (2017): “¿Cómo se puede mejorar la competitividad de las cooperativas agroalimentarias?", CIRIEC-España, Revista de Economía Pública, Social y Cooperativa, 90, 103-121. DOI: 10.7209/CIRIEC- E.89.8854. 
LÓPEZ, E. (2015): "A cadea láctea en Galicia: retos e estratexias nun mercado liberalizado", Cadernos do Consello Económico e Social de Galicia, 5, 5-9.

MELIÁ, E. \& MARTÍNEZ, A. (2015): "Factores críticos del éxito de una fusión de cooperativas", ITEA, Información Técnica Económica Agraria Revista de la Asociación Interprofesional para el Desarrollo Agrario (AIDA), 111, 73-92.

MELIÁ, E. \& PERÍS, M. (2017): "Los procesos de integración de las cooperativas agroalimentarias. De la norma a la realidad. Especial referencia a la Ley 13/2013 de Fomento de la Integración Cooperativa", REVESCO, Revista de Estudios Cooperativos, 126, 177-197. DOI: 10.5209/ reve.58614.

MELIÁ, E. \& SERVER, R.J. (2002): "Bases y parámetros económico- sociales de la integración en cooperativas agrarias . Caso estudio del proceso de fusión Bases y parámetros económico-sociales de la integración en cooperativas agrarias. Caso estudio del proceso de fusión", CIRIEC-España, Revista de Economía Pública, Social y Cooperativa, 41, 85-110.

MONZÓN, J.L. (2012): "Las cooperativas ante la globalización: magnitudes, actividades y tendencias", Ekonomiaz, Revista Vasca de Economía, 79 (01), 13-30.

MOUGÁN, H. (Dir.) (2014): XVIII Informe sobre a xestión e o estado económico do cooperativismo agrario en Galicia, Asociación de Cooperativas Agrarias - AGACA (Ed.) (1st ed.).

PRESIDENCIA DE LA XUNTA DE GALICIA (1998): Ley 5/1998, de 18 de diciembre, de cooperativas de Galicia.

SERVER, R.J. \& MELIÁ, E. (2002): "La concentración empresarial en cooperativas agrarias. Formulación de un modelo económico para los acuerdos de fusión", Estudios Agrosociales y Pesqueros, 196, 33-52.

SIEBEL, S. (2016): Cooperative economies in a global age, Doctoral Thesis, RMIT University.

SINEIRO, F., LÓPEZ, E. \& SANTISO BLANCO, J.A. (2017): "Dinámica recente e retos de futuro do sector lácteo en Galicia. Cuestións para o debate". En: Fundación Juana de Vega de 1 de decembro de 2017, 1-13. 